\title{
Do educational interventions aimed at nurses to support the implementation of evidence-based practice improve patient outcomes? A systematic review
}

\begin{abstract}
Background: Numerous articles have sought to identify the impact of educational interventions for improving evidence-based practice (EBP) amongst nurses, most of these focus on skills and knowledge acquired. No systematic review has explored whether this educational input translates into improved patient outcomes.

Objectives: To review the evidence on (1) The change in patient outcomes following educational interventions to support practising nurses in implementing EBP. (2) The instruments or methods used to determine whether EBP education improves patient outcomes.

Methods: A systematic review following PRISMA guidance was conducted. Literature was comprehensive searched including 6 databases, journal handsearching, citation tracking, and grey literature websites. Studies were included if they reported an EBP educational intervention aimed at practising nurses and contained objective or selfreported measures of patient related outcomes. The quality of the included studies was assessed using a modified Health Care Practice R\&D Unit (HCPRDU) tool. Because of the poor homogeneity of the included studies, the data were analysed by narrative synthesis.
\end{abstract}

Results: Of the 4,284 articles identified, 18 were included: 12 pre-post studies, three qualitative studies, and three mixed-methods study designs. The level of quality was modest in the studies. The results of the EBP educational interventions on patient outcomes were assessed using three methods: individual projects to implement an evidence-based approach, qualitative approaches, and a questionnaire survey. The majority of the articles concluded there was a positive change in patient outcomes following an educational intervention to improve EBP; a wide range of context specific 
outcomes were described.

Conclusion: Educational interventions for clinical nurses to support the implementation of EBP show promise in improving patient outcomes. However, the direct impact of EBP interventions on clinical outcomes is difficult to measure. Further testing and development is needed to improve the quality of studies and evaluation instruments in order to confirm the current findings.

Key words: evidence-based practice; EBP; clinical nurses; patient outcomes; instruments

\section{Introduction}

The implementation of Evidence-Based Practice (EBP) in health care has been advocated for many years to bridge the gap between science and practice (Crabtree, Brennan, Davis, \& Coyle, 2016). This is aligned to the 2020 goal of the Institute of Medicine (IOM) that $90 \%$ of clinical decisions be evidence-based to improve the quality of health care (Olsen, Goolsby, \& McGinnis, 2009). Clinical nurses, working on the frontline of health care, are in a key position to question practice and use the most up-to-date research evidence in health care decision making (Cullen \& Titler, 2004). Furthermore, Yost, Ciliska, and Dobbins (2014) also highlighted that finding ways to promote the use of scientific evidence in nursing care is paramount, because nurses are the largest group of health care professionals. However, the translation of evidence into the clinical setting is still limited (Saunders \& Vehvilainen-Julkunen, 2016). Lack of adequate EBP knowledge and skills in practising nurses has been identified as an important barrier to achieving change (Melnyk, Fineout-Overholt, Gallagher-Ford, \& Kaplan, 2012; Saunders \& Vehvilainen-Julkunen, 2016). To address this, there have been numerous studies exploring educational interventions to encourage nurses to adopt and implement EBP within routine nursing practice (Balakas, Sparks, Steurer, \& Bryant, 2013; Connor, Dwyer, \& Oliveira, 2016; Green et al., 2014; Milne, Krishnasamy, Johnston, \& Aranda, 2007). 
Previous systematic reviews on educational interventions for promoting EBP with practising clinicians have focused on identifying changes in EBP knowledge and skills (Haggman-Laitila, Mattila, \& Melender, 2016), multidisciplinary health care professionals such as physicians, nurses, physiotherapists, psychologists, and medical students (Fiander et al., 2015; Hines, Ramsbotham, \& Coyer, 2015; Young, Rohwer, Volmink, \& Clarke, 2014), pedagogical strategies (Aglen, 2016; Phillips et al., 2014; J. Yost et al., 2014), and the instruments for evaluating EBP education (Shaneyfelt et al., 2006). For example, Young et al. (2014) synthesised 16 systematic reviews that evaluated the effectiveness of teaching EBP to multidisciplinary health professionals. The review included a total of 81 separate studies, and the evidence showed that conducting training programmes could lead to improvements in EBP attitude, skills, and knowledge across health professions. Furthermore, Phillips et al. (2014) investigated the methods to report educational interventions for developing EBP knowledge and skills, but most of the included articles were from the field of medicine. Haggman-Laitila et al. (2016) reviewed studies relating to practicing nurses but also focussed on the benefits of education on improving participants' EBP knowledge and skills.

Knowing that EBP education improves knowledge and skills does not tell us whether this knowledge is actually implemented in practice or whether EBP education results in improved patient care or outcomes. There is a lack of review evidence exploring whether an EBP educational intervention results in the implementation of an evidence-based approach and improved patient outcomes. This review therefore focuses on the effectiveness of EBP educational interventions in relation to improving patient outcomes within nursing practice. Firstly, changing practice and improving patient care are the ultimate purposes of implementing EBP educational programmes (Balakas et al., 2013). Staff nurses need to translate those EBP knowledge and skills into "real-world" settings and clinical practice. However, the effective implementation of EBP in routine care is often challenged by various barriers, including lack of time, lack of cultural and organisational support, competing priorities, and workloads 
(Melnyk et al., 2012). Secondly, the clinical environment is complex, and although nurses may have adequate EBP knowledge (Suemoto et al., 2015) it may not always be possible to successfully translate scientific evidence into nursing practice. Measuring or evaluating whether this change has been implemented is complex as it is difficult to attribute changes in patient care or outcomes to the EBP intervention itself and little is known about how best to measure these changes. As noted above, several reviews have identified that educational programmes can develop key skills for undertaking EBP such as searching and synthesising literature but limited data exists on whether conducting EBP training can change behaviour or patient outcomes (Straus, Ball, Balcombe, Sheldon, \& McAlister, 2005). This may be because changes in knowledge and skills are easier to measure than the implementation of a change on patient outcomes. Educators need instruments to evaluate and document the programmatic impact of their teaching programmes (Shaneyfelt et al., 2006). Given the complexity of evaluating the effect of educational interventions to support the implementation of EBP, it is essential to provide guidance for EBP educators by highlighting suitable methods or instruments to assess patient outcomes and to make recommendations for education based on the current evaluation science. Identification of current instruments or methods to evaluate patient outcomes could help educators to understand the effects of EBP education and determine whether new methods are needed to measure the impact of EBP education on patient care.

Therefore, with a focus on practising nurses, this systematic review aimed to gather, assess, and synthesise the current available evidence on the effects of EBP education on patient outcomes. The research questions for this review were: (1) What patient outcomes have been achieved following educational interventions to support practising nurses in implementing EBP? (2) What instruments or methods have been used to determine whether EBP education improves patient outcomes?

\section{Methods}

\subsection{Design}


The PRISMA checklist (2009) guided the review methods. A protocol (available on request) was agreed at the outset amongst two of the authors (YW and AB). A PICOS statement (PRISMA, 2009) guided the search and inclusion of articles. This involved the participants $(\mathrm{P})$ practising nurses, the intervention (I) any education relating to evidence-based practice, the comparison $(\mathrm{C})$ other or no education, the outcome $(\mathrm{O})$ would be patient outcomes (see below) and S any study design would be considered as the effects of educational interventions are not easily measured, particularly when the outcomes may not be immediately apparent or occur in a complex environment (Moore et al, 2015).

\subsection{Search strategy}

In May 2017, the following databases were searched with no date restrictions: Medline (Ovid), Web of Science, CINAHL, the Cochrane Library, ERIC, and LISA. Searches were conducted from the inception of each database to May 2017 (Table S1). In line with guidance relating to searching, additional methods were adopted to improve the comprehensiveness of the searches (Brettle and Grant, 2004; Stansfield et al, 2016; Hopewell et al, 2007; Hartling et al, 2017). This included manual checking of reference lists of the retrieved articles, citation tracking, and the content tables of four key journals (Worldviews on Evidence-Based Nursing, Nurse Education Today, Implementation Science, and the Journal of Continuing Education in Nursing). To identify grey literature OpenSIGLE (http://www.computescotland.com/opensigle-theopen-sesame-to-european-grey-literature-1429.php), Open Grey (http://www.opengrey.eu/), the Grey Literature Report by the New York Academy of Medicine Library (http://www.greylit.org/), and the ClinicalTrials.gov (https://clinicaltrials.gov/) was searched.

\subsection{Inclusion and exclusion criteria}

The following criteria were developed to determine which studies would be eligible for inclusion in the review: (1) Studies that reported an educational intervention using the 
EBP process and principles. (2) The participants were clinical nurses, defined as advanced practice nurses or registered nurses (including nurse practitioners or clinical nurse specialists) (Jennifer et al., 2014). Studies of multidisciplinary EBP training on practising healthcare professionals, provided that outcomes for professional groups, such as nurses, were reported separately. (3) Contained objective or self-reported measures of patient related outcomes in the following areas: health status measures; disease incidence, duration or cure rates; mortality; complication rates; readmission rates; adherence rates; satisfaction; continuity of care; use of resources (e.g. cost-benefit analyses) (Reeves, Perrier \& Goldman, 2013). (4) Studies written in English. (5) Any study design (6) No limitations or restrictions on year of publication were applied. Studies published as a conference abstract, or where the full text could not be obtained after contacting the authors were excluded.

\subsection{Study Selection}

The PRISMA flow diagram was used to guide the study selection process. The titles and abstracts of all the located studies were screened by two reviewers, according to the inclusion and exclusion criteria. The full text of those deemed relevant were screened again by two reviewers (YW and JO) who independently selected the articles using a standard study selection form, based on the inclusion and exclusion criteria. Disagreements were resolved by consensus or the third person (AB).

\subsection{Assessment of methodological quality}

The Health Care Practice R\&D Unit (HCPRDU) evaluation tools were adopted to assess the quality of the included studies (Long, 2003; Long, Godfrey, Randall, Brettle, $\&$ Grant, 2002a, 2002b). These were selected because the research designs of the included studies in the review are diverse and the HCPRDU evaluation tools can be used to assess any type of research evidence by providing a descriptive summary of methodological quality for quantitative, qualitative, and mixed-methods studies. To provide a summary of the quality of each study, a scoring criterion was developed for each element of the study design (Aveyard, 2014). 0 points ("many limitations"), 1 point 
(“some limitations"), and 2 points (“excellent”) Haggman-Laitila et al. (2016). A maximum 12 points was possible for quantitative or qualitative studies (0-6 = "many limitations", 7-8 = "some limitations", and 9-12= "excellent") and 14 points for the mixed method $(0-6$ points $=$ "many limitations", 7-11 = "some limitations", and $12-$ 14 points = "excellent"). The summary quality score of each paper is noted in Table S2 and the conclusions were drawn on the results of all the papers regardless of quality, as they were difficult to compare.

\subsection{Data extraction and synthesis}

Two reviewers (YW and JO) independently extracted the data from each of the included articles based on a standard data extraction form. The form was based on the PICOS components of the review that included general information (author, publication year and setting/location), the participants' characteristics (sample size, sample selection, profession, job experience, and previous EBP exposure), the intervention (aim of the study, education duration, content, material/model used, and method of delivery), outcomes (patient outcomes and evaluation method), and the study design. When information was missing, one reviewer (YW) contacted the authors to request further data. If additional data could not be obtained, the reviewer coded the question as "Not reported".

The wide variety of interventions, the inconsistency of outcome measures, and incomplete data meant that the extracted data were unable to be combined for metaanalysis. Therefore, the extracted information was analysed by narrative synthesis.

\section{Results}

\subsection{Description and quality of the studies}

\subsubsection{Search results}

A total of 4,284 articles were identified for screening, 15 of which were identified 
through reference and citation tracking. Of the 4,284 studies, 394 duplicates were excluded. Then, the titles and abstracts of the remaining 3,890 articles were screened, and 3,829 were excluded according to the inclusion and exclusion criteria of the review. Furthermore, the remaining 61 articles, which could not be determined by the title and abstract alone, were assessed by the full text. Of the 61 full-text studies, 43 were excluded and 18 were included in the systematic review: 12 quantitative articles, three qualitative studies, and three mixed-methods study designs. The PRISMA flow diagram of this process is shown in Figure 1.

\subsubsection{Description of the included studies}

Eighteen studies met the inclusion criteria and were included in the systematic review, including 12 quantitative articles (Bruheim, Woods, Smeland, \& Nortvedt, 2014; Crabtree et al., 2016; Cullen, Titler, \& Rempel, 2011; Gawlinski \& Becker, 2012; Green et al., 2014; Hockenberry, Brown, Walden, \& Barrera, 2009; Jack, Roberts, \& Wilson, 2003; Sciarra, 2011; Selig \& Lewanowicz, 2008; Steurer, 2010; Weeks, Moore, \& Allender, 2011; Wells, Free, \& Adams, 2007), three qualitative studies (Balakas et al., 2013; Christenbery, Williamson, Sandlin, \& Wells, 2016; Milne et al., 2007), and three mixed-methods study designs (Connor et al., 2016; Cullen \& Titler, 2004; Friesen, Brady, Milligan, \& Christensen, 2017). Descriptions of these studies are presented in Table S2. The majority of the studies took place in the USA, one was in the UK, one Australia and one in Norway. The EBP interventions were wide ranging. All covered the EBP process and principles, some used a didactic approach, others used workshops, mentors or a project in practice or a combination of approaches.

\subsubsection{Quality appraisal of the included studies}

The quality of the 18 studies was assessed using the HCPRDU evaluation tools and the scoring system developed for the purpose of this review. Five of the studies were assessed as "excellent", seven studies could be classified as having "some limitations", 
and six as having "many limitations" (Table S2). It was not always possible to determine the sample size for each study.

\subsection{Patient Outcomes of the EBP interventions}

Three methods were identified which sought to link the effectiveness of an EBP educational intervention to patient outcomes. This included: the implementation of an individual project using EBP principles and evaluating the subsequent change on patient outcomes; the use of interviews to provide qualitative evidence describing changes in patient outcomes, and a questionnaire survey (Table S2).

\subsubsection{Patient outcomes following the implementation of EBP projects}

Changes in patient outcomes were evaluated using a wide variety of EBP projects. All the 18 studies required or encouraged participants to conduct an EBP project at the end of the programme, 16 of which described the project-related patient outcomes in the paper. The description of the project results varied from a short mention to detailed presentations. Each project had a different focus or aim; of the 16 articles, 15 reported positive patient outcomes of EBP projects, and the remaining one reported mixed outcomes.

Some papers reported specific patient outcomes for a range of clinical areas. These improvements included positive changes in pain management (Green et al., 2014; Hockenberry et al., 2009; Wells et al., 2007), the rates of urinary catheter utilisation (Green et al., 2014), the rates of pressure ulcers (Green et al., 2014), the length of stay in hospital (Cullen \& Titler, 2004; Green et al., 2014; Milne et al., 2007), the number of calls to the outpatient orthopaedic clinic (Green et al., 2014), the infection rate of dialysis catheters (Steurer, 2010), the rates of infections in relation to central line in neonatal intensive care unit (NICU) patients (Balakas et al., 2013), cost (Cullen \& Titler, 2004), anxiety of patients (Cullen \& Titler, 2004), the rates of aspiration pneumonia (Crabtree et al., 2016), and the rates of ventilator-associated pneumonia (Wells et al., 2007). For example, in Cullen and Titler's (2004) study, one of the projects 
implemented an EBP-based sedation protocol in the Medical Intensive Care Unit, which resulted in decreasing costs by $\$ 450,000$ and the length of stay in the first year after implementation.

Four studies stated that conducting EBP projects in clinical practice improved patient outcomes but did not report specific details. Furthermore, one study reported mixed results regarding patient outcomes: data was collected on 11 projects conducted by nurses, six of these found adequate evidence to change clinical practice whereas the other five projects found insufficient or no evidence to answer the question (Milne et al., 2007).

\subsubsection{Patient outcomes based on qualitative data}

Four studies included qualitative data to describe the patient outcomes. Data was collected using focus groups with 65 clinical nurses. For example, in the study conducted by Connor et al. (2016), one of the nurses highlighted specifically how this EBP programme influenced patient outcomes: this program promoted us to adopt oral medication which has no narcotic effect.

\subsubsection{Patient outcomes based on questionnaire survey}

One study identified patient outcomes through a questionnaire survey (Cullen et al., 2011). Firstly, a total of 417 nurses that attended 27 programmes between 2003 and 2009 completed an evaluation of the immediate effects of the programme. As a result of the programme $99.8 \%$ of the nurses reported that they had identified strategies to implement EBP changes in clinical practice. To evaluate the long-term impact of the programme, 94 questionnaires were sent to participants between 1-5 years after completing the programme. Of these 33 were returned. $30 \%$ of these had completed their EBP projects in a clinical setting, and $42 \%$ were in the process. Furthermore, $79 \%$ of nurses followed up over the long term reported that the programme had resulted in 
better patient outcomes, which included decreasing risk, enhancing symptom management, reducing hospitalisation, and improving satisfaction.

\subsection{Instruments or methods for evaluating patient outcomes}

The instruments or methods used to assess the effectiveness of the EBP interventions in relation to improving patient outcomes were most commonly based on EBP projects and qualitative interviews. Of the 18 studies, 12 were based on the project-specific outcomes of the EBP programme participants' projects. As noted above, the projects were varied, thus the data collected also varied and depended on the project in question. For example, a project which sought to reduce catheterization would collect data on the rates of urinary catherization at the beginning and end of the project. Similarly, if a project sought to decrease length of stay, data on length of stay before and after implementation would be studied. Little or no detail was provided on the appropriateness or quality of the methods used to collect the data or whether there were any confounding variables. Three studies used focus group interviews to gather details regarding the impact on patient outcomes, once the training programme was complete, and two combined the two methods to assess patient outcomes. Furthermore, only one study used two self-designed questionnaires as the instruments to carry out the assessment (Cullen et al., 2011). One questionnaire with three sections was used to evaluate the immediate post-programme effects. Then, another questionnaire was designed to test the long-term impact of the programme and comprised five sections. Notably, the validity and reliability of these two self-reported questionnaires was not reported in the paper.

\section{Discussion}

\subsection{Summary of main results}

This systematic review is the first to explore whether providing educational 
interventions on evidence-based practice translates into improved patient outcomes in nursing practice. Although based on evaluation projects and qualitative data, the results suggest that positive changes on patient outcomes can be made following the implementation of specific evidence-based approaches (or projects).

The majority of the impacts of EBP interventions on patient care were found in a wide range of specific outcomes such as pain, the length of stay in hospital, cost, the rates of pneumonia, as well as other clinical areas. Positive experiences from participants were also identified during qualitative interviews (Table S2). The above findings complement Haggman-Laitila et al. (2016), who found that educational interventions on clinical nurses were effective in elevating nurses' EBP knowledge and skills and Yost et al. (2014), who concluded that implementing knowledge translation strategies on nurses to support the use of research evidence in clinical settings can benefit patient outcomes. This is in contrast to a review conducted by Fiander et al. (2015), who found that interventions to promote the use of electronic health information by health care practitioners contribute no effects to clinical practice or patient outcomes, though it does suggest that the use of electronic health information increases. The diversity of the populations and interventions are likely to be a proposed reason for the inconsistency in the results of these systematic reviews. For example, Selig and Lewanowicz (2008) highlighted that the skill set required for EBP should be different for an experienced clinical nurse and a student nurse. This was also the reason why we determined to conduct a review specifically in EBP educational interventions on clinical nurse populations. The results of our review showed that EBP educational activities for clinical nurses have promising results on patient outcomes.

However, though positive outcomes have been identified in the review, some of the improved outcomes are based on nurses' verbal reports and insights through qualitative data and questionnaire surveys. This does not provide a robust evidence base demonstrating objective changes in patient outcomes and may be subject to bias from both nurses' and patients' insights. 


\subsection{The difficulties in measuring the impact of EBP education on patient outcomes}

Exploring the gap between EBP educational input and patient outcomes has been recommended for many years, although it is challenging to measure such benefits reliably, as confirmed in this review. Weightman and Williamson (2005) highlighted that identifying the benefits for clinical outcomes to managers, funding bodies, and users is an essential way to persuade them to become the champions of EBP education, because as Harrison and Beraquet (2010) warned, "value and worth" is what matters to the majority of stakeholders. This is in line with Balakas et al. (2013), who stated that some managers did not support staff nurses to join EBP fellowship programmes because they appeared to misunderstand the EBP process and the benefits for patient outcomes. Furthermore, Connor et al. (2016) and Bruheim et al. (2014) also recommended that there is a need to make sure that EBP knowledge and skills can actual be applied in nursing practice to improve patient outcomes. The key difficulties include tracking the direct impact of an intervention which is remote from clinical care (Brettle et al., 2011; Shaneyfelt et al., 2006) and the variety of interventions and potential patient outcomes (Table S2) which make comparisons between studies impossible. For example, the majority of specific clinical outcomes in the review were identified based on EBP projects that the participants had completed following an educational intervention. The projects in one study ranged from urinary catheter utilisation to interventions for low birth weight infants to cast care pathways. Capturing patient outcomes from these diverse projects and attributing them to one intervention highlight the difficulties in finding a robust, "one evaluation model fits all" approach to assess the effects of the interventions.

It is therefore perhaps not surprising that the articles reviewed mainly used descriptive quasi-experimental pre-post surveys (12/18 studies), qualitative (3/18 articles), and mixed-methods study designs (3/18 articles) to evaluate the value of EBP programmes for patient outcomes. According to the scoring system developed to complement the critical appraisal tools, 13 of the included studies could be assessed as having "some limitations" or "many limitations", and five as "excellent". Although 
these methods may be appropriate to establish links, they cannot demonstrate cause and effect and do not provide high quality research evidence to confirm the current findings. This is in line with previous systematic reviewers who highlighted that there is an absence of "high quality" experimental design research to evaluate the value of EBP interventions (Brettle et al., 2011; Haggman-Laitila et al., 2016; Wagner \& Byrd, 2004). Although descriptive and qualitative research is also important when little is known about a field Melnyk (2012) highlighted that there are few rigorously designed comparative effectiveness studies and RCTs to determine the effects of EBP interventions in speeding the translation of this evidence into real-world settings.

One way forward may be to recognise that EBP education can only make a contribution in a wide range of clinical outcomes and can only be measured indirectly according to improving the quality of care of clinicians (Jackson, 2004). This review demonstrates that EBP project-based assessment and qualitative interviews perhaps are two appropriate methods to begin to evaluate the patient outcomes of EBP educational interventions. Although it is evident that there is still room to improve data collection methods on the clinical impacts of EBP education as these methods are unlikely to be recognised by the EBP movement as high-quality evidence. One potential approach would be to follow the MRC guidance on evaluating complex interventions (Moore et al, 2015) and use this as a basis for more robust study design. Additionally, Reeves et al (2013) examined inter-professional education and its impact on patient outcomes and located a number of robust studies which may also offer some ideas for improving methodological quality. Using these kinds of approaches would help bring the measurement of EBP educational interventions into line with the high quality of research expected by those espousing evidence-based practice.

One component of this improvement in methodological quality would be to develop more standardised tools to evaluate the effects of EBP interventions on patient care. Currently, the majority of instruments with reasonable validity and reliability for testing education in EBP are focused on nurses' EBP knowledge, attitude, and skills (Shaneyfelt et al., 2006), which are easier to measure. The evidence from this review, 
however could be used to develop more standardised tools on the potential patient outcomes that could be achieved, but these would need to be validated before wider use, and they may still be insufficient in capturing a wide range of outcomes over the longer term.

\subsection{Limitations of this review}

Some limitations have been identified in the systematic review. Firstly, because of the poor homogeneity of the study designs, EBP interventions, outcome measurements, and incomplete data, it was not possible to pool the majority of the indexes for a metaanalysis to draw a conclusion of the study. Secondly, the language of the included articles was limited to English due to time and the challenges of translation, which may lead to some bias of the review. Thirdly, four included studies failed to provide complete data on the patient outcomes from conducting EBP projects, even after direct requests to the authors (Christenbery et al., 2016; Friesen et al., 2017; Gawlinski \& Becker, 2012; Weeks et al., 2011). Moreover, inconsistent indexes, such as the characteristics of the included studies and description of the EBP educational intervention and patient outcomes, might have caused some bias in the results of the review. All these may have led to the omission of some essential data on EBP education as an intervention in clinical nurses to improve patient outcomes, leading to some potential bias in the results.

\section{Conclusions}

In conclusion, this review provided evidence that conducting EBP educational interventions on nurses in clinical practice can have a positive impact on patient outcomes, which can demonstrate the usefulness and importance of such programmes. However, the direct impact of EBP interventions on clinical outcomes is difficult to measure because of the wide range of potential outcomes which are difficult to attribute to the results of the intervention itself. Capturing process data following the implementation of EBP projects and qualitative interviews have been identified as two 
main approaches to evaluate patients' outcomes to date. Furthermore, this review has also concluded that there is a dearth of high-level experimental research to assess patient outcomes in EBP educational programmes. Future researchers are urged to determine if experimental designs will be suitable to test the impact of EBP interventions on patients and develop standardised instruments to evaluate direct patient outcomes.

\section{Conflict of Interest}

No conflict of interest has been declared by the authors.

\section{Supporting Information}

Table S1. Search strategy.

Table S2. Description of the studies in the review.

\section{References}

Aglen, B. 2016. Pedagogical strategies to teach bachelor students evidence-based practice: A systematic review. Nurse Educ Today, 36, 255-263. doi: 10.1016/j.nedt.2015.08.025

Aveyard, H. 2014. Doing a literature review in health and social care: A practical guide: McGrawHill Education (UK).

Balakas, K., Sparks, L., Steurer, L., \& Bryant, T. 2013. An outcome of evidence-based practice education: Sustained clinical decision-making among bedside nurses. Journal of pediatric nursing, 28(5), 479-485.

Brettle, A., \& Grant, M. J. (2004). Finding the evidence for practice: a workbook for health professionals: Edinburgh: Churchill Livingstone.

Brettle, A., Maden-Jenkins, M., Anderson, L., McNally, R., Pratchett, T., Tancock, J., . . W Webb, A. 2011. Evaluating clinical librarian services: a systematic review. Health Information \& Libraries Journal, 28(1), 3-22.

Brettle, A., Maden, M., \& Payne, C. 2016. The impact of clinical librarian services on patients and health care organisations. Health Information \& Libraries Journal, 33(2), 100-120.

Bruheim, M., Woods, K. V., Smeland, S., \& Nortvedt, M. W. 2014. An educational program to transition oncology nurses at the Norwegian Radium Hospital to an evidence-based practice model: development, implementation, and preliminary outcomes. $J$ Cancer Educ, 29(2), 224-232. doi: 10.1007/s13187-013-0575-9 
Christenbery, T., Williamson, A., Sandlin, V., \& Wells, N. 2016. Immersion in Evidence-Based Practice Fellowship Program: A Transforming Experience for Staff Nurses. J Nurses Prof Dev, 32(1), 15-20. doi: 10.1097/nnd.0000000000000197

Connor, L., Dwyer, P., \& Oliveira, J. 2016. Nurses' Use of Evidence-Based Practice in Clinical Practice After Attending a Formal Evidence-Based Practice Course: A Quality Improvement Evaluation. Journal for nurses in professional development, 32(1), E1-E7.

Crabtree, E., Brennan, E., Davis, A., \& Coyle, A. 2016. Improving Patient Care Through Nursing Engagement in Evidence-Based Practice. Worldviews Evid Based Nurs, 13(2), 172-175. doi: 10.1111/wvn.12126

Cullen, L., \& Titler, M. G. 2004. Promoting evidence-based practice: an internship for staff nurses. Worldviews Evid Based Nurs, 1(4), 215-223. doi: 10.1111/j.1524-475X.2004.04027.x

Cullen, L., Titler, M. G., \& Rempel, G. 2011. An advanced educational program promoting evidence-based practice. West J Nurs Res, 33(3), 345-364. doi: 10.1177/0193945910379218

Dearholt, S., \& Dang, D. 2012. Johns Hopkins nursing evidence-based practice: Models and guidelines. Sigma Theta Tau.

Fiander, M., McGowan, J., Grad, R., Pluye, P., Hannes, K., Labrecque, M., . . . Tugwell, P. 2015. Interventions to increase the use of electronic health information by healthcare practitioners to improve clinical practice and patient outcomes. The Cochrane Library(3), 1-52. doi: 10.1002/14651858.CD004749.pub3

Friesen, M. A., Brady, J. M., Milligan, R., \& Christensen, P. 2017. Findings From a Pilot Study: Bringing Evidence-Based Practice to the Bedside. Worldviews on Evidence-Based Nursing, 14(1), 22-34.

Gawlinski, A., \& Becker, E. 2012. Infusing research into practice: a staff nurse evidence-based practice fellowship program. J Nurses Staff Dev, 28(2), 69-73. doi: 10.1097/NND.0b013e31824b418c

Green, A., Jeffs, D., Huett, A., Jones, L. R., Schmid, B., Scott, A. R., \& Walker, L. 2014. Increasing capacity for evidence-based practice through the evidence-based practice academy. The journal of continuing education in nursing, 45(2), 83-90.

Haggman-Laitila, A., Mattila, L. R., \& Melender, H. L. 2016. Educational interventions on evidencebased nursing in clinical practice: A systematic review with qualitative analysis. Nurse Educ Today, 43, 50-59. doi: 10.1016/j.nedt.2016.04.023

Harrison, J., \& Beraquet, V. 2010. Clinical librarians, a new tribe in the UK: roles and responsibilities. Health Information \& Libraries Journal, 27(2), 123-132.

Hartling, L., Featherstone, R., Nuspl, M., Shave, K., Dryden, DM., Vandermeer, B.2017. Grey literature in systematic reviews: a cross-sectional study of the contribution of non-English reports, unpublished studies and dissertations to the results of meta-analyses in childrelevant reviews. BMC Medical Research Methodology, 17(1):64. doi: 10.1186/s12874-0170347-z.

Hines, S., Ramsbotham, J., \& Coyer, F. 2015. The effectiveness of interventions for improving the research literacy of nurses: a systematic review. Worldviews on Evidence-Based Nursing, 12(5), 265-272.

Hockenberry, M., Brown, T., Walden, M., \& Barrera, P. 2009. Teaching evidence-based practice skills in a hospital. $J$ Contin Educ Nurs, 4Q(1), 28-32. 
Hopewell, S., Clarke, M. J., Lefebvre, C., \& Scherer, R. W. (2007). Handsearching versus electronic searching to identify reports of randomized trials. The Cochrane Library.

Jack, B. A., Roberts, K. A., \& Wilson, R. W. 2003. Developing the skills to implement evidence based practice - a joint initiative between education and clinical practice. Nurse Educ Pract, 3(2), 112-118. doi: 10.1016/s1471-5953(02)00108-7

Jackson, C. 2004. How do I measure the impact of my service? (Guideline). (p:210-222). In: BOOTH, A and BRICE, A.(eds) Evidence-based practice for information professionals: a handbook. London: Facet.: Cambridge University Press.

Long, A. 2003. HCPRDU: Evaluation tool for quantitative studies. University of Salford Health Care Practice Research and Development Unit. Retrieved April 30, 2008.

Long, A., Godfrey, M., Randall, T., Brettle, A., \& Grant, M. 2002a. HCPRDU Evaluation tool for mixed methods studies.

Long, A., Godfrey, M., Randall, T., Brettle, A., \& Grant, M. 2002b. HCPRDU evaluation tool for qualitative studies.

Melnyk, B. M. 2012. The Role of Technology in Enhancing Evidence-Based Practice, Education, Heathcare Quality, and Patient Outcomes: A Call for Randomized Controlled Trials and Comparative Effectiveness Research. Worldviews on Evidence-Based Nursing, 9(2), 63-65.

Melnyk, B. M., Fineout-Overholt, E., Gallagher-Ford, L., \& Kaplan, L. 2012. The state of evidencebased practice in US nurses: Critical implications for nurse leaders and educators. Journal of Nursing Administration, 42(9), 410-417.

Milne, D. J., Krishnasamy, M., Johnston, L., \& Aranda, S. 2007. Promoting evidence-based care through a clinical research fellowship programme. Journal of clinical nursing, 16(9), 16291639. doi: 10.1111/j.1365-2702.2006.01748.x

Olsen, L., Goolsby, W., \& McGinnis, J. 2009. Institute of Medicine (US), Roundtable on Evidence based Medicine. Leadership commitments to improve value in health care: finding common ground workshop summary: Washington, DC: National Academies Press.

Phillips, A. C., Lewis, L. K., McEvoy, M. P., Galipeau, J., Glasziou, P., Hammick, M., ... Williams, M. T. 2014. A systematic review of how studies describe educational interventions for evidencebased practice: stage 1 of the development of a reporting guideline. BMC Med Educ, 14, 152. doi: 10.1186/1472-6920-14-152

Reeves, S., Perrier, L., Goldman, J., Freeth, D., \& Zwarenstein, M. (2013). Interprofessional education: effects on professional practice and healthcare outcomes (update). The Cochrane Library.

Sackett, D. L. 2000. Evidence-based medicine: how to practice and teach. EBM.

Saunders, H., \& Vehvilainen-Julkunen, K. 2016. The state of readiness for evidence-based practice among nurses: An integrative review. Int J Nurs Stud, 56, 128-140. doi: 10.1016/j.jinurstu.2015.10.018

Sciarra, E. 2011. Impacting practice through evidence-based education. Dimensions of Critical Care Nursing, 30(5), 269-275.

Selig, P. M., \& Lewanowicz, W. 2008. Translation to Practice Developing an Evidence-Based Practice Nurse Internship Program. AACN advanced critical care, 19(3), 325-332.

Shaneyfelt, T., Baum, K. D., Bell, D., Feldstein, D., Houston, T. K., Kaatz, S., . . Green, M. 2006. Instruments for evaluating education in evidence-based practice: a systematic review. JAMA, 296(9), 1116-1127. doi: 10.1001/jama.296.9.1116 
Stansfield, C., Dickson, K., \& Bangpan, M. (2016). Exploring issues in the conduct of website searching and other online sources for systematic reviews: how can we be systematic?. Systematic reviews, 5(1), 191.

Steurer, L. M. 2010. An evidence-based practice scholars program: one institution's journey toward excellence. J Contin Educ Nurs, 41(3), 139-143. doi: 10.3928/00220124-20100224-04

Stevens, K. R. 2004. ACE Star Model of EBP: knowledge transformation. Academic Center for Evidence-based Practice. The University of Texas Health Science Center at San Antonio, 1-3.

Straus, S. E., Ball, C., Balcombe, N., Sheldon, J., \& McAlister, F. A. 2005. Teaching evidence-based medicine skills can change practice in a community hospital. Journal of General Internal Medicine, 20(4), 340-343. doi: 10.1111/j.1525-1497.2005.04045.x

Suemoto, C. K., Ismail, S., Correa, P., Zaina, F., Jerves, T., Pesantez, L., . . Fregni, F. 2015. Five-year review of an international clinical research-training program. Advances in Medical Education and Practice, 6, 249-257. doi: 10.2147/amep.s66627

Wagner, K. C., \& Byrd, G. D. 2004. Evaluating the effectiveness of clinical medical librarian programs: a systematic review of the literature. Journal of the Medical Library Association, 92(1), 14.

Weeks, S. M., Moore, P., \& Allender, M. 2011. A regional evidence-based practice fellowship: Collaborating competitors. Journal of Nursing Administration, 41(1), 10-14.

Weightman, A., \& Williamson, J. 2005. The value and impact of information provided through library services for patient care: a systematic review. Health Information \& Libraries Journal, 22(1), 4-25.

Wells, N., Free, M., \& Adams, R. 2007. Nursing research internship: enhancing evidence-based practice among staff nurses. J Nurs Adm, 37(3), 135-143. doi: 10.1097/01.NNA.0000262732.14123.a2

Yost, J., Ciliska, D., \& Dobbins, M. 2014. Evaluating the impact of an intensive education workshop on evidence-informed decision making knowledge, skills, and behaviours: a mixed methods study. BMC Med Educ, 14(1), 13.

Yost, J., Thompson, D., Ganann, R., Aloweni, F., Newman, K., McKibbon, A., . . Ciliska, D. 2014. Knowledge Translation Strategies for Enhancing Nurses' Evidence-Informed Decision Making: A Scoping Review. Worldviews Evid Based Nurs, 11(3), 156-167. doi: 10.1111/wvn.12043

Young, T., Rohwer, A., Volmink, J., \& Clarke, M. 2014. What are the effects of teaching evidencebased health care $(\mathrm{EBHC})$ ? Overview of systematic reviews. PloS one, 9(1), e86706. 